\section{Epizyme connects the DOTs}

\section{By Joanne Kotz, Senior Editor}

Epizyme Inc. has reported the first in vivo data for one of the histone methyltransferase DOT1L inhibitors identified at the company, showing the compound increased overall survival in a mouse model of mixedlineage leukemia. ${ }^{1}$ According to the company, the data provide proof of concept for targeting this class of epigenetic enzymes. After selecting a clinical candidate, Epizyme intends to file an IND and take DOT1L inhibitors into the clinic in this leukemia.

Mixed-lineage leukemia (MLL) is an aggressive subset of acute leukemias with an annual incidence of about 4,000 patients in the U.S and Europe. Chemotherapy or human stem cell transplant, the standard treatments, are often ineffective.

The disease is characterized by chromosomal translocations of the gene encoding the histone methyltransferase myeloid-lymphoid or mixed-lineage leukemia (MLL; HRX). These rearrangements lead to the formation of various MLL fusion proteins, which recruit a second histone methyltransferase, DOT1L. This recruitment of DOT1L triggers oncogenic overexpression of multiple leukemogenic genes.

Previous research had shown that DOT1L is essential for the development of MLL. ${ }^{2}$

"There is a clear mechanistic rationale for a DOT1L inhibitor in MLL," said Robert Copeland, EVP and CSO of Epizyme.

"A number of genetic studies have pointed to an essential role for the histone methyltransferase DOT1L in the proliferation of acute myelogenous leukemias transformed by MLL fusion proteins. DOT1L has therefore been seen as an excellent target for the specific targeting of this subgroup of AMLs," added Kristian Helin, CSO of EpiTherapeutics ApS.

EpiTherapeutics is developing small molecule inhibitors of histone methyltransferases and histone demethylases.

Using the crystal structure of DOT1L and the chemical structure of the enzyme's substrate as guides, Epizyme designed EPZ004777. In vitro, the small molecule inhibited DOT1L with an $\mathrm{IC}_{50}$ of $400 \mathrm{pM}$ and showed at least 1,000-fold selectivity for DOT1L over 9 other histone methyltransferases. In MLL cell lines, EPZ004777 decreased expression of leukemogenic genes targeted by the MLL oncogenic fusion protein and inhibited proliferation compared with vehicle controls.

Epizyme, along with academic collaborators at the Dana-Farber Cancer Institute, Children's Hospital Boston and Harvard Medical School, next tested the inhibitor in animals. In a mouse xenograft model of MLL, continuous infusion of EPZ004777 increased overall survival compared with vehicle infusion.

Data were published in Cancer Cell.

"It's the first evidence for in vivo efficacy for any histone methyltransferase inhibitor," said Copeland. "While the data have important implications for DOT1L in MLL, we also think the data have far-reaching implications for the entire class. We think these data portend success for the entire strategy of targeting histone methyltransferases.”

\section{Moving toward the clinic}

According to Copeland, Epizyme has identified compounds that are "significant improvements" over EPZ004777. "We have a number of inhibitors of DOT1L that are likely good enough to go into the clinic, and we are in the late stages of final characterization," he said.

The company has not provided a timeline for an IND filing. However, Copeland did say that the company is "progressing aggressively to the clinic."

One question is whether a clinical candidate can block the target for a sufficient duration with an acceptable therapeutic window.

Patrick Trojer, director of biology at Constellation Pharmaceuticals Inc., said extended inhibition by histone methyltransferase inhibitors might be necessary to achieve a therapeutic benefit. He noted that data in the paper show a lag time of days before the DOT1L inhibitor has an effect on target gene expression and cell proliferation.

He added that these results are consistent with Constellation's experience with inhibiting certain histone methyltransferases. Constellation is targeting histone methyltransferases, histone demethylases and bromodomain-containing proteins.

The results in the paper suggest toxicity might be an issue, said Helin, who also is director of the Biotech Research \& Innovation Centre at the University of Copenhagen. The mouse data show inhibiting DOT1L is "not overly toxic," but the DOT1L inhibitor did lead to increases in the number of white blood cells in a short-term assay. "Therefore, unfortunately long-term treatment might lead to more severe side effects," he added.

Copeland countered that "the hematologic effects are rather minor, and with the improved compounds we are pursuing for clinical applications we remain confident in our ability to achieve an attractive therapeutic index."

\section{Outside MLL}

Epizyme also is exploring the potential of DOT1L inhibitors outside of MLL. "We are empirically evaluating the inhibitor in other cancer types," said Copeland.

"There's not a clear genetic link that we know of yet to cancers other than MLL," said Scott Armstrong, a coauthor on the paper and a coleader of the leukemia program at Dana-Farber. 
He did say the hox genes, which are regulated by the MLL protein, are highly expressed in 30\%-40\% of acute myelogenous leukemias (AMLs) and in many other cancers. Although it has not yet been demonstrated, DOT1L might be playing a role, suggested Armstrong, who also is an associate professor in the Department of Pediatrics at Harvard Medical School and in hematology/oncology at Children's Hospital Boston.

Epizyme has filed patent applications covering composition of matter on its DOT1L inhibitors.

Kotz, J. SciBX 4(30); doi:10.1038/scibx.2011.841

Published online Aug. 4, 2011

\section{REFERENCES}

1. Daigle, S.R. et al. Cancer Cell; published online July 12, 2011; doi:10.1016/j.ccr.2011.06.009

Contact: Roy M. Pollock, Epizyme Inc., Cambridge, Mass. e-mail: rpollock@epizyme.com

2. Nguyen, A.T. \& Zhang, Y. Genes Dev. 25, 1345-1358 (2011)

COMPANIES AND INSTITUTIONS MENTIONED

Children's Hospital Boston, Boston, Mass.

Constellation Pharmaceuticals Inc., Cambridge, Mass.

Dana-Farber Cancer Institute, Boston, Mass.

EpiTherapeutics ApS, Copenhagen, Denmark

Epizyme Inc., Cambridge, Mass.

Harvard Medical School, Boston, Mass.

University of Copenhagen, Copenhagen, Denmark 